\title{
Assessment of the intake of dietary fibre from cereal foods: an epidemiological approach
}

\author{
ALAN J. SILMAN \\ From the Department of Clinical Epidemiology, London Hospital Medical College
}

SUMMARY In a study evaluating the intake of dietary fibre from cereal foods the results obtained from a standard 7-day weighed record were compared with simpler methods. This showed that weighing was unnecessary to obtain a valid measure of intake. There was also a low ratio of within-subject to between-subject variation: thus four days of recording are all that are required. The 4-day unweighed record is cheaper, less complex, and may be expected to produce higher completion rates than the 7-day weighed record.

The methodological problems of dietary surveys are frequently overlooked when hypotheses of diet and disease are tested and this can lead to erroneous conclusions. The selection of the most suitable method for a particular study requires that the objectives of that study be clearly defined. ${ }^{1}$ Dietary surveys tend to have one of two objectives:

(i) The study of diet in groups classified by non-dietary criteria, for example, age, sex, presence or absence of disease.

(ii) The classification of individuals into a particular dietary group, for example, by thirds of a distribution of intakes, and then comparison between the groups of the presence of a disease or a disease-related variable.

Achieving the second objective requires a method which must be capable of distinguishing the intake of individuals and therefore recall and interview methods, useful in distinguishing between groups, are not precise enough. ${ }^{2} \mathrm{~A}$ method appropriate for classifying individuals must ensure a valid representation of the food consumed and it must also take account of the individual daily variation. Absolute validity in assessing current consumption is impossible to achieve in free-living conditions; the aim is to discover a method with a high and defined degree of validity relative to a recognised standard method. This in practice is the 'weighed inventory method' whereby the subject weighs his cooked food and the record is analysed using food tables.

There is also an individual daily variation in intake of all dietary factors and allowance must be made for this to ensure correct classification of individuals to a particular dietary group. If too few measurements of a dietary factor are obtained for an individual, it may then be impossible to distinguish him from anothe with respect to diet. $^{3}$ The larger the ratio off $\dot{\omega}$ within-person to between-person variation, the $i$ greater the number of days' intake which should beo studied. ${ }^{4}$

A 7-day weighed record is possibly at the limit of what may be achieved with suitable co-operation.? Such a method is complex, costly in financial and human resources, and often has a low acceptability Thus a compromise must be reached between the feasible and the desirable and the method chosefo will, in large part, depend on the characteristics of the nutrient studied.

Dietary fibre from cereal sources (DCF) is a nutrient of increasing medical interest. Its main sources are few and can be described in terms of standard 'household' measures, for example, one slice from a standard cut loaf. These can then be converted to estimated weights the accuracy of which can be ascertained. Such an approach could not be used for other nutrients, for example, fat, because there are no equivalent 'household' measures.

Similarly, the individual daily variation in DCF is likely to be small compared with other nutrients. For many individuals, a significant proportion of the daily intake of DCF is consumed at breakfast, and breakfast patterns within individuals probably remain fairly constant. Specifically, this study tested two possibilities:

(i) that an unweighed record is adequate for estimating an individual's daily intake of DCF.

(ii) that a period shorter than seven days would be adequate for distinguishing the DCF intakes of different individuals. 


\section{Material and methods}

The subjects were male, aged $45-64$, all working in the same factory (May and Baker Limited, Dagenham, Essex). This factory had been included in the Heart Disease Prevention Project (HDPP), St. Mary's Hospital, London, and the employees agreed to take part in the present study. Twelve volunteers representing all grades of employment were selected. The sample was not random; the selection method adopted was based on two factors. Firstly, a weighed survey requires a large degree of voluntary co-operation. Secondly, the main concern of this study was the comparison of different survey methods within the same individual.

The method was adapted from one previously described for weighed surveys. ${ }^{6}$ Each man was interviewed separately and the nature of the study explained. The men were encouraged to follow their normal food patterns and no particular reference was made to the inclusion of cereal foods. They were asked to weigh and record all items of food and drink consumed over a 7-day continuous period, and also to record the amounts of all items, using a standard household measure or a description of portion size. A booklet of blank record forms was issued, together with a written set of instructions repeating what was said during the interview. At the end of the 7-day period, the men were interviewed again and their records checked for completeness.

A table of weight equivalents to standard household portions was constructed for most common foods. This was based on extensive previous dietary research (Marr, unpublished data) supplemented by sample weighings of currently available breads and breakfast cereals. Each record was analysed twice:

(a) using the description of size only for those foods with a cereal content. The estimated weight equivalent was coded together with a code number relating the food to standard food composition tables; ${ }^{*}$ (b) using the actual weights of the foods eaten which were coded using the method in (a).

Cereal foods only were analysed from the unweighed data, because such data would not be suitable for assessing the intake of other dietary variables, for example, calories or total fats.

The records were analysed by computer using a programme incorporating food tables data on the composition of foods ${ }^{7}$ supplemented by information on dietary fibre. ${ }^{8}$ The programme also enabled the dietary fibre from different sources to be calculated.

\footnotetext{
- Used with kind permission of Miss Jean Marr.
}

\section{Results}

All 12 men originally interviewed agreed to participate and 11 produced a satisfactory record; the man whose record was rejected was unable to weigh his food because of extensive travelling away from home. The remaining records, yielding a total of 77 man-days of recording, were analysed.

The mean daily intake of DCF for each man, calculated from the weighed record, is shown in the Table. The individual means were all within two standard deviations from the group mean with the intakes probably following a normal distribution.

Table Intake of dietary cereal fibre by subject. Mean daily intake in grams/day derived from a 7-day weighed record

\begin{tabular}{lrl}
\hline Subject number & Mean daily intake & $S D$ \\
\hline 1 & 7.38 & 3.18 \\
2 & 11.79 & 4.23 \\
3 & 9.43 & 4.91 \\
4 & 14.03 & 2.91 \\
5 & 6.40 & $2 \cdot 54$ \\
6 & 4.52 & 0.48 \\
7 & 6.64 & 2.84 \\
8 & 12.77 & 3.88 \\
9 & 8.77 & 4.08 \\
10 & 8.24 & 1.60 \\
11 & 11.09 & 2.95 \\
\hline
\end{tabular}

Mean of subject means $=9 \cdot 19$ SD 2.95

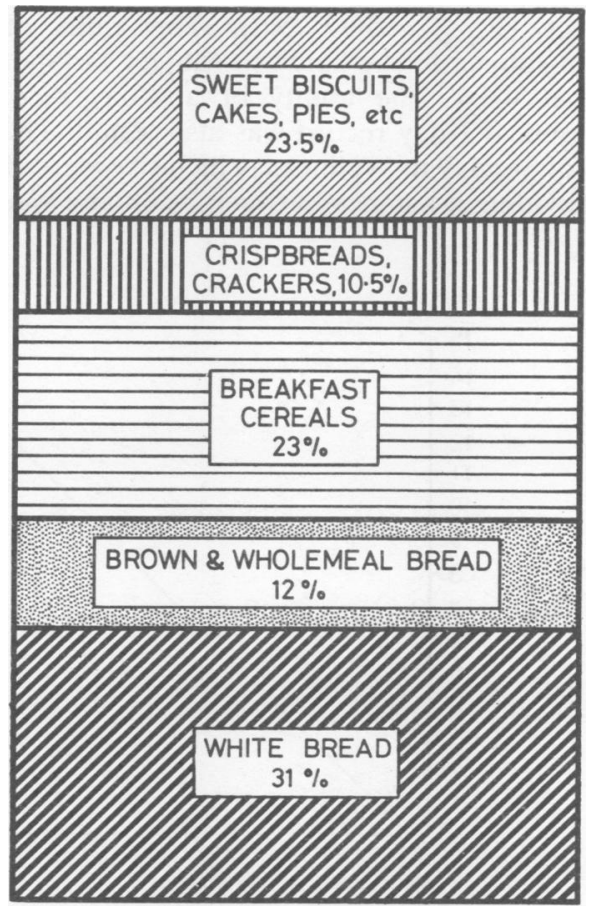

Total dietary cereal fibre $=100 \%$

Fig. 1 Sources of dietary cereal fibre. 
The sources of DCF were also analysed (Fig. 1). Brown and wholemeal breads, containing two and three times respectively the amount of DCF as white bread, were a minor source in comparison with the volume of white bread consumed. Total bread consumption as a whole, though, was a poor predictor of total DCF intake $(r=-0.57 \mathrm{NS})$. This accords with the results from previous studies. ${ }^{9}$

The relationship of the values obtained for DCF from the unweighed to the values from the weighed record (Fig. 2) showed a very strong correlation between the two. Most cereal foods, as well as containing fibre, are also major sources of carbohydrate and thus contribute significantly to the total calorie intake. It has been suggested that in studies considering the role of DCF in disease ${ }^{10}$ the intake of DCF should be expressed as grams per 1000 calories of total dietary intake. This did not alter the findings, because a high correlation was observed between the total intake of DCF and the intake expressed as grams DCF per 1000 calories $(r=+0.94 P<0.001)$.

There was considerable variation in the intakes of DCF recorded (Fig. 3). The sources of this variation were analysed using an analysis of variance table. There was a highly significant variation between subjects $\left(F_{10,60}=5.66 \mathrm{P}<0.001\right)$; but no significant variation between different recordings within a subject $\left(F_{6,60}=0.32 \mathrm{NS}\right)$. The ratio of the within-subject to the between-subject variation was low, at $0 \cdot 16$. The ability of a 4-day record to represent a 7-day record was also tested. The four days of Sunday to Wednesday inclusive were chosen as providing a balance between week and weekend days.

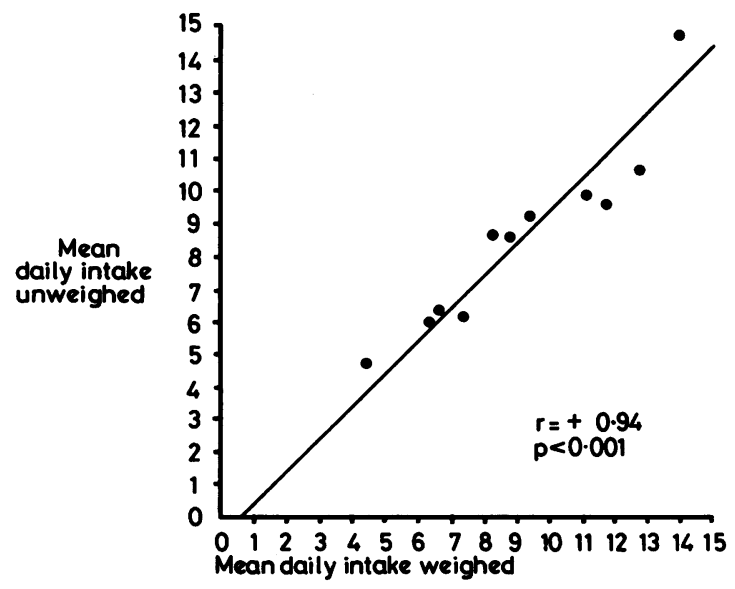

Fig. 2 Intakes of dietary cereal fibre in grams/day. Weighed compared with unweighed diet.

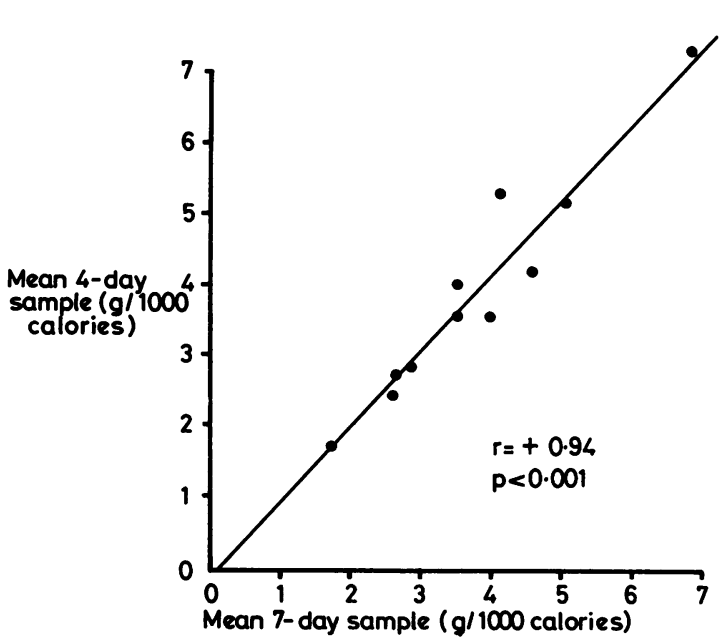

Fig. 3 Dietary cereal fibre intake 1000 calories mean daily intake: 7-day weighed sample compared with 4-day weighed sample.

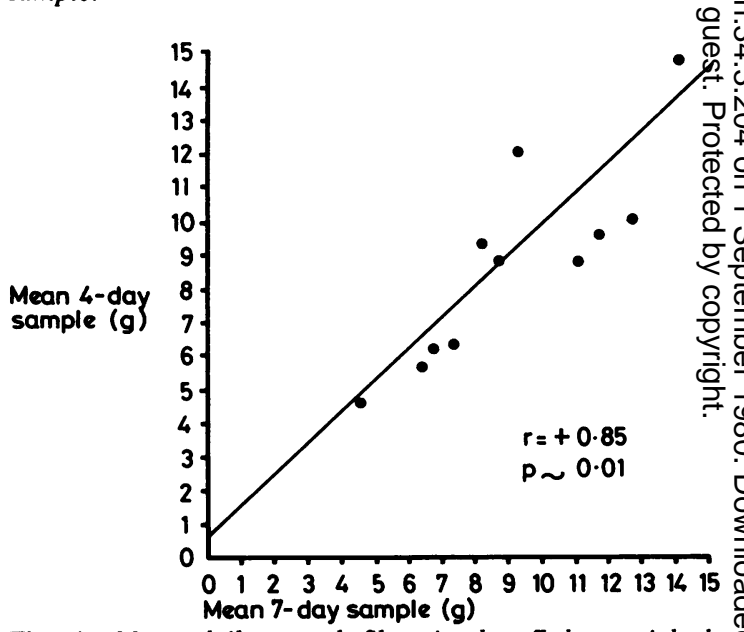

Fig. 4 Mean daily cereal fibre intake: 7-day weighed sample compared with 4-day unweighed sample.

A strong correlation was observed between the mean intake of DCF calculated from the 7-day weighed record and that calculated from the 4-day unweighed record (Fig. 4). This strong correlation persisted when the 7-day (weighed) mean intake of DCF, expressed as grams per 1000 calories, was compared with the 4-day (unweighed) mean $(r=+0.94 P<0.001)$.

\section{Discussion}

This study, although small, had a high completion rate and high acceptability.

The main sources of DCF are few and the amount eaten can be estimated from an accurate description 
of portion size. The result is useful in indicating that weighed records are not required in epidemiological surveys of DCF intake. Thus cost and complexity are reduced and acceptability may be expected to increase. $^{2}$

The ratio of within-subject to between-subject variation, at $0 \cdot 16$, was low compared with the results from studies of other dietary factors; for example, for dietary cholesterol it is $3 \cdot 0^{3}$ and for total calories it is $0 \cdot 91 .^{5}$ In the only study of DCF (Marr and Heady, unpublished data) a higher ratio was obtained than in the present study, and the authors calculated that four days of weighed recording would be necessary to classify individuals by thirds of intake of DCF, with an $80 \%$ chance of correctly classifying the top and bottom third and only a $1 \%$ chance of gross misclassification. In this study it has been demonstrated that a 4-day unweighed record would also prove an acceptable substitute for the standard 7-day record, thereby reducing the period of investigation.

Patterns of consumption of individual nutrients vary, and a methodology suitable for studying one nutrient is not necessarily the most appropriate for another. DCF is a useful model in the epidemiological study of diets, but it can be applied only to other nutrients with sources that are few and easily quantifiable, and with low inter-individual variation. In this study I have concentrated on dietary fibre from cereal sources and the conclusions obtained are not applicable to dietary fibre from fruit and vegetables where the pattern of consumption is quite different.

I thank Professor Geoffrey Rose and Dr Richard Heller for permission to study subjects from the Heart Disease Prevention Project, Dr Jean Cuthbert at May and Baker and the men who participated, Miss Margaret Tebble for programming, and
Professor J. N. Morris, Dr. Austin Heady, and especially Miss Jean Marr, for their helpful comments and advice at all stages. The study was performed as part of the requirements for MSc Soc Med (London University) and was financially supported by the South-east Thames Regional Health Authority.

Reprints from Dr. A. J. Silman, Department of Clinical Epidemiology, London Hospital Medical College, Turner Street, London E1 2AD.

\section{References}

${ }^{1}$ Young CM. Comparison of results of dietary surveys made by different methods. Proceedings of the 4th International Congress of Dietetics Stockholm 1965: 119-26.

${ }^{2}$ Marr JW. Individual dietary surveys: purposes and methods. World Rev Nutr Diet 1971; 13: 105-64.

${ }^{3}$ Liu K, Stamler J, Dyer A, McKeever J, McKeever P. Statistical methods to assess and minimise the role of intra-individual variability in obscuring the relationship between dietary lipids and serum cholesterol. J Chronic Dis 1978; 31: 399-418.

'Heady JA. The classification of individuals in large-scale dietary surveys. PhD thesis. London: University of London, 1961.

${ }^{5}$ Gardner MJ, Heady JA. Some effects of within-person variability in epidemiology studies. J Chronic Dis 1973; 26: 781-95.

${ }^{6}$ Marr JW. Individual weighed dietary surveys. Nutrition 1965; 19: 18-24.

${ }^{7}$ Paul AA, Southgate DAT. In: Mc Cance RA, Widdowson ED, ed. The Composition of Foods. 4th edn. London: HMSO, 1978.

${ }^{8}$ Southgate DAT, Bailey B, Collinson E, Walker AF. A guide to calculating intakes of dietary fibre. J Hum Nutr 1976; 30: 303-13.

${ }^{9}$ Morris JN, Marr JW, Clayton DG. Dietary fibre from cereals and the incidence of coronary heart disease. $J$ Plant Foods 1978; 3: 45.

${ }^{10}$ Morris JN, Marr JW, Clayton DG. Diet and heart: a postscript. Br Med J 1977; ii: 1307-14. 